\title{
THE DETERMINANTS OF THE THREE-BAND BLOCK MATRICES
}

\author{
Grzegorz Biernat, Sylwia Lara-Dziembek, Edyta Pawlak \\ Institute of Mathematics, Czestochowa University of Technology \\ Czestochowa, Poland \\ grzegorz.biernat@im.pcz.pl,sylwia.lara@im.pcz.pl,edyta.pawlak@im.pcz.pl
}

\begin{abstract}
In the paper the method of calculating of the determinants of block matrices is presented. The three-band matrices are considered, both in the particular case (3D) as well as in the general case.
\end{abstract}

\section{Introduction}

When we attempt to solve many issues connected with heat flow or with the theory of vibrations the system of equations which reading to the band matrix are obtained. Typically, these systems are solved using the numerical methods. This work is an introduction to solve these systems using algebraic methods.

\section{Solution of the problem}

Let us consider the following matrices:

- the three-band matrix

$$
A_{1}=\left[\begin{array}{rrrrrrr}
a & 1 & \ldots & \ldots & \ldots & \ldots & \ldots \\
1 & a & 1 & \ldots & \ldots & \ldots & \ldots \\
\vdots & \vdots & \vdots & \ddots & \vdots & \vdots & \vdots \\
\ldots & \ldots & \ldots & \ldots & 1 & a & 1 \\
\ldots & \ldots & \ldots & \ldots & \ldots & 1 & a
\end{array}\right]_{n_{1} \times n_{1}}
$$

and

- the three-band block matrix 


$$
A_{k}=\left[\begin{array}{rrrrrrr}
A_{k-1} & I_{k-1} & \ldots & \ldots & \ldots & \ldots & \ldots \\
I_{k-1} & A_{k-1} & I_{k-1} & \ldots & \ldots & \ldots & \ldots \\
\vdots & \vdots & \vdots & \ddots & \vdots & \vdots & \vdots \\
\ldots & \ldots & \ldots & \ldots & I_{k-1} & A_{k-1} & I_{k-1} \\
\ldots & \ldots & \ldots & \ldots & \ldots & I_{k-1} & A_{k-1}
\end{array}\right]_{n_{k} \times n_{k}}, k \geq 2
$$

So, the block matrices in 2D and in 3D case are presented in these form

$$
\begin{aligned}
A_{2} & =\left[\begin{array}{rrrrrrr}
A_{1} & I_{1} & \ldots & \ldots & \ldots & \ldots & \ldots \\
I_{1} & A_{1} & I_{1} & \ldots & \ldots & \ldots & \ldots \\
\vdots & \vdots & \vdots & \ddots & \vdots & \vdots & \vdots \\
\ldots & \ldots & \ldots & \ldots & I_{1} & A_{1} & I_{1} \\
\ldots & \ldots & \ldots & \ldots & \ldots & I_{1} & A_{1}
\end{array}\right]_{n_{2} \times n_{2}} \\
A_{3} & =\left[\begin{array}{rrrrrrr}
A_{2} & I_{2} & \ldots & \ldots & \ldots & \ldots & \ldots \\
I_{2} & A_{2} & I_{2} & \ldots & \ldots & \ldots & \ldots \\
\vdots & \vdots & \vdots & \ddots & \vdots & \vdots & \vdots \\
\ldots & \ldots & \ldots & \ldots & I_{2} & A_{2} & I_{2} \\
\ldots & \ldots & \ldots & \ldots & \ldots & I_{2} & A_{2}
\end{array}\right]_{n_{3} \times n_{3}}
\end{aligned}
$$

etc.

It is easy to check that the determinant of the block matrix $A_{1}$ is as follows [1]

$$
\operatorname{det} A_{1}=a^{n_{1}}-\left(\begin{array}{c}
n_{1}-1 \\
1
\end{array}\right) a^{n_{1}-2}+\left(\begin{array}{c}
n_{1}-2 \\
2
\end{array}\right) a^{n_{1}-4}-\ldots
$$

Then, following the same way we obtain the determinant of matrix $A_{2}$

$$
\operatorname{det} A_{2}=\operatorname{det}\left[A_{1}^{n_{2}}-\left(\begin{array}{c}
n_{2}-1 \\
1
\end{array}\right) A_{1}^{n_{2}-2}+\left(\begin{array}{c}
n_{2}-2 \\
2
\end{array}\right) A_{1}^{n_{2}-4}-\ldots\right]
$$

Assuming the polynomial of degree $n_{2}$

$$
\begin{aligned}
& f_{2}\left(x_{1}\right)=x_{1}^{n_{2}}-\left(\begin{array}{c}
n_{2}-1 \\
1
\end{array}\right) x_{1}^{n_{2}-2}+\left(\begin{array}{c}
n_{2}-2 \\
2
\end{array}\right) x_{1}^{n_{2}-4}-\ldots= \\
& =\left(x_{1}-p_{1,1}\right)\left(x_{1}-p_{1,2}\right)\left(x_{1}-p_{1,3}\right) \cdot \ldots \cdot\left(x_{1}-p_{1, n_{2}}\right)
\end{aligned}
$$

where $p_{1, j}$ are zeros of the polynomial $f$, for $j=1, \ldots, n_{2}$, 
we obtain the determinant of the three-band block matrix

$$
\begin{aligned}
& \operatorname{det} A_{2}=\operatorname{det}\left[\left(A_{1}-p_{1,1} I_{1}\right)\left(A_{1}-p_{1,2} I_{1}\right)\left(A_{1}-p_{1,3} I_{1}\right) \cdot \ldots \cdot\left(A_{1}-p_{1, n_{1}} I_{1}\right)\right]= \\
& =\underbrace{\operatorname{det}\left(A_{1}-p_{1,1} I_{1}\right)}_{W_{A_{1}}\left(p_{1,1}\right)} \cdot \underbrace{\operatorname{det}\left(A_{1}-p_{1,2} I_{1}\right)}_{W_{A_{1}}\left(p_{1,2}\right)} \cdot \underbrace{\operatorname{det}\left(A_{1}-p_{1,3} I_{1}\right)}_{W_{A_{1}}\left(p_{1,3}\right)} \cdot \ldots \cdot \underbrace{\operatorname{det}\left(A_{1}-p_{1, n_{1}} I_{1}\right)=}_{W_{A_{1}}\left(p_{1, n_{1}}\right)} \\
& =\left(\lambda_{1}-p_{1,1}\right)\left(\lambda_{1}-p_{1,2}\right)\left(\lambda_{1}-p_{1,3}\right) \cdot \ldots \cdot\left(\lambda_{1}-p_{1, n_{1}}\right) \cdot \\
& \cdot\left(\lambda_{2}-p_{1,1}\right)\left(\lambda_{2}-p_{1,2}\right)\left(\lambda_{2}-p_{1,3}\right) \cdot \ldots \cdot\left(\lambda_{2}-p_{1, n_{1}}\right) \cdot \\
& \cdot \ldots \cdot \\
& \cdot\left(\lambda_{n_{1}}-p_{1,1}\right)\left(\lambda_{n_{1}}-p_{1,2}\right)\left(\lambda_{n_{1}}-p_{1,3}\right) \cdot \ldots \cdot\left(\lambda_{n_{1}}-p_{1, n_{1}}\right)
\end{aligned}
$$

where $\lambda_{1}, \ldots, \lambda_{n_{1}}$ are eigenvalues of the matrix $A_{1}$ and $W_{A_{1}}\left(p_{1,1}\right), \ldots, W_{A_{1}}\left(p_{1, n_{1}}\right)$ are the values of the characteristic polynomials $W_{A_{1}}$ of the matrix $A_{1}[2,3]$.

Moreover, the determinant of the matrix $A_{3}$ is given by the formula

$$
\operatorname{det} A_{3}=\operatorname{det}\left[A_{2}^{n_{3}}-\left(\begin{array}{c}
n_{3}-1 \\
1
\end{array}\right) A_{2}^{n_{3}-2}+\left(\begin{array}{c}
n_{3}-2 \\
2
\end{array}\right) A_{2}^{n_{3}-4}-\ldots\right]
$$

and then applying the polynomial of degree $n_{3}$

$$
\begin{aligned}
& f_{2}\left(x_{2}\right)=x_{2}^{n_{3}}-\left(\begin{array}{c}
n_{3}-1 \\
1
\end{array}\right) x_{2}^{n_{3}-2}+\left(\begin{array}{c}
n_{3}-2 \\
2
\end{array}\right) x_{2}^{n_{3}-4}-\ldots= \\
& =\left(x_{2}-p_{2,1}\right)\left(x_{2}-p_{2,2}\right)\left(x_{2}-p_{2,3}\right) \cdot \ldots \cdot\left(x_{2}-p_{2, n_{3}}\right)
\end{aligned}
$$

we obtain

$$
\begin{aligned}
& \operatorname{det} A_{3}=\operatorname{det}\left[\left(A_{2}-p_{2,1} I_{2}\right)\left(A_{2}-p_{2,2} I_{2}\right)\left(A_{2}-p_{2,3} I_{2}\right) \cdot \ldots \cdot\left(A_{2}-p_{2, n_{2}} I_{2}\right)\right]= \\
& =\underbrace{\operatorname{det}\left(A_{2}-p_{2,1} I_{2}\right)}_{W_{A_{2}}\left(p_{2,1}\right)} \cdot \underbrace{\operatorname{det}\left(A_{2}-p_{2,2} I_{2}\right)}_{W_{A_{2}}\left(p_{2,2}\right)} \cdot \underbrace{\operatorname{det}\left(A_{2}-p_{2,3} I_{2}\right)}_{W_{A_{2}}\left(p_{2,3}\right)} \cdot \cdots \cdot \underbrace{\operatorname{det}\left(A_{2}-p_{2, n_{2}} I_{2}\right)=}_{W_{A_{2}}\left(p_{2, n_{2}}\right)} \\
& =\underbrace{\operatorname{det}\left[A_{1}-\left(p_{1,1}+p_{2,1}\right) I_{1}\right]}_{W_{A_{1}}\left(p_{1,1}+p_{2,1}\right)} \cdot \underbrace{\operatorname{det}\left[A_{1}-\left(p_{1,1}+p_{2,2}\right) I_{1}\right]}_{W_{A_{1}}\left(p_{1,1}+p_{2,2}\right)} \cdot \cdots \cdot \underbrace{\operatorname{det}\left[A_{1}-\left(p_{1,1}+p_{2, n_{2}}\right) I_{1}\right]}_{W_{A_{1}}\left(p_{1,1}+p_{2, n_{2}}\right)} .
\end{aligned}
$$




$$
\begin{aligned}
& \text { '... } \\
& \underbrace{\cdot \operatorname{det}\left[A_{1}-\left(p_{1, n_{1}}+p_{2,1}\right) I_{1}\right]}_{W_{A_{1}}\left(p_{1, n_{1}}+p_{2,1}\right)} \cdot \underbrace{\operatorname{det}\left[A_{1}-\left(p_{1, n_{1}}+p_{2,2}\right) I_{1}\right]}_{W_{A_{1}}\left(p_{1, n_{1}}+p_{2,2}\right)} \cdot \cdots \cdot \underbrace{\operatorname{det}\left[A_{1}-\left(p_{1, n_{1}}+p_{2, n_{2}}\right) I_{1}\right]}_{W_{A_{1}}\left(p_{1, n_{1}}+p_{2, n_{2}}\right)}
\end{aligned}
$$

Generally, continuing above algorithm we have

$$
\operatorname{det} A_{k}=\operatorname{det}\left(A_{k-1}^{n_{k}}-r_{k-1,1} A_{k-1}^{n_{k}-2}+r_{k-1,2} A_{k-1}^{n_{k}-4}-\ldots\right)
$$

Assuming

$$
\operatorname{det} A_{k}=\operatorname{det}\left[A_{k-1}^{n_{k}}-\left(\begin{array}{c}
n_{k}-1 \\
1
\end{array}\right) A_{k-1}^{n_{k}-2}+\left(\begin{array}{c}
n_{k}-2 \\
2
\end{array}\right) A_{k-1}^{n_{k}-4}-\ldots\right]
$$

and

$$
\begin{aligned}
& f_{k}\left(x_{k-1}\right)=x_{k-1}^{n_{k}}-\left(\begin{array}{c}
n_{k}-1 \\
1
\end{array}\right) x_{k-1}^{n_{k}-2}+\left(\begin{array}{c}
n_{k}-2 \\
2
\end{array}\right) x_{k-1}^{n_{k}-4}-\ldots= \\
& =\left(x_{k-1}-p_{k-1,1}\right)\left(x_{k-1}-p_{k-1,2}\right)\left(x_{k-1}-p_{k-1,3}\right) \cdot \ldots \cdot\left(x_{k-1}-p_{k-1, n_{k}}\right)
\end{aligned}
$$

we obtain

$$
\operatorname{det} A_{k}=\underbrace{\operatorname{det}\left[A_{1}-\left(p_{1,1}+p_{2,1}+\ldots+p_{k-1,1}\right) I_{1}\right]}_{W_{A_{1}}\left(p_{1,1}+p_{2,1}+\ldots+p_{k-1,1}\right)} \ldots
$$

The above expression includes $n_{1} \times n_{2} \times \ldots \times n_{k}$ factors.

In conclusion, the determinant of the three-band block matrix is given by the product of the respective characteristic polynomials of matrix $A_{1}$.

The determinants of the block matrices are applicable among others in problems of heat transfer. For example, the Finite Differences Method (FDM) for the Fourier equation (in the case of the internal nodes) leads to the seven-band internal equations in each time step and then the matrix of the system equations is a threeband block matrix.

\section{References}

[1] Biernat G., Boryś J., Całusińska I., Surma A., The three - band matrices, Scientific Research of the Institute of Mathematics and Computer Science 2008, 2(7), 119-122.

[2] Lang S., Algebra, Springer Science Business Media Inc., 2002.

[3] Mostowski A., Stark M., Elementy algebry wyższej, PWN, Warszawa 1975. 